\title{
The Impact of Adjuvant Chemotherapy Initiation Time on the Outcome of Breast Cancer
}

\section{Md. Nesreen Ahmed Mosalam*, Md. Hany Mohamed Abd El Aziz, Amr S Saad and Asmaa Wahid Mohamed}

Faculty of Medicine, Ain Shams University, Cairo, Egypt

\begin{abstract}
Importance: It is well established that adjuvant chemotherapy yielded better prognosis in breast cancer. Yet, there is a wide discrepancy in the allowed time frame between surgery and chemotherapy.

Purpose: To identify the optimal initiation time to chemotherapy (TTC) after definitive surgery for breast cancer patients in terms of overall survival (OS) and disease-free survival (DFS), also to identify the main causes of chemotherapy delay at department of Clinical Oncology and Nuclear Medicine at Ain Shams University Hospitals.

Patients and methods: A retrospective study including a population of 300 patients with non-metastatic breast cancer received adjuvant chemotherapy at time interval Between January 2007 till December 2011 using database from the department of Clinical Oncology and Nuclear Medicine, Ain Shams University Hospital. Chi-Square test and Kaplan-Meier method were used.

Results: Among the studied population, the mean age at diagnosis was 50 years and the median TTC was 5 weeks. Compared with patients receiving chemotherapy earlier than 4 weeks from definitive surgery, there was no adverse outcome as regards the OS and DFS among those with TTC of 4 to 8 weeks, 8 to 12 weeks or more than 12 weeks with non-significant $P$ values $(0.67$ and 0.9 , respectively). Factors associated with chemotherapy delay included sociodemographic problems among rural patients, long residential distance to institution, system related causes as late referral, governmental insurance support and long waiting lists for diagnostic imaging.
\end{abstract}

Conclusion: Adjuvant chemotherapy is equally effective up to 12 weeks after definitive surgery with similar survival benefit among breast cancer patients even those with high risk factors.

\section{Mini Abstract}

Adjuvant chemotherapy is equally effective up to 12 weeks with similar survival benefit in breast cancer patients in a retrospective study included 300 patients.

Keywords: Adjuvant chemotherapy; Breast cancer; Oncology; Surgery

\section{Introduction}

Breast cancer is globally considered the most common cancer diagnosed among women accounting for $23 \%$ of all cancers [1].

In Egypt, breast cancer is the most frequent cancer among females. It accounts for $38.8 \%$ of all cancers in women [2].

No doubts regarding the benefit of adjuvant chemotherapy in indicated breast cancer patients as proved by many large randomized clinical trials conducted by the early breast cancer trialists' collaborative group but these chemotherapy regimens were accredited based only on the time frame applied in the trial obtained its efficacy [3].

Developing countries lack of financial issues has mainly been the obstacle causing delay in diagnosis and treatment. Other patient related factors as lack of knowledge of breast cancer, denial, late presentation or trying to access to an affordable medical care [4].

Although the impact of treatment delay after diagnosis in cancer is poorly defined, it is well known that early diagnosis and treatment yield better prognosis [5].

There is a wide discrepancy in the allowed time between surgery and chemotherapy as the TTC ranged from 2-5 weeks according to The National Surgical Adjuvant Breast and Bowel Project trials and the landmark CMF trial conducted by Bonadonna et al. [6] after following up the patients for 20 years.
According to the National Breast and Cervical Cancer Early Detection Program (NBCCEDP) benchmark, the time frame between identification of any suspicious mammogram screening test and diagnosis should not exceed 60 days and maximum another 60 days till initiation of treatment [7].

While the time frame recommended by the ASCO and NCCN organization's quality measures falls within 120 days of diagnosis especially for young women aged less than 70 years with non-metastatic breast cancer [8].

A recent ASCO study was conducted on the overall survival, relapse-free survival and distant free survival outcomes according to TTC over 6,827 patients showing that there is indifferences in terms of OS and DFS between different cohorts starting chemotherapy within 0 to up to more than 60 days postoperatively but it showed a significance only with more advanced stage disease who experienced worse outcome in terms of RFS and DRFS (stage II and III) and OS

*Corresponding author: Md. Nesreen Ahmed Mosalam, Faculty of Medicine, Ain Shams University, Cairo, Egypt, Tel +201224465406, Fax: +224346606; E-mail: dr.nesreen2000@gmail.com

Received June 03, 2017; Accepted June 22, 2017; Published June 29, 2017

Citation: Mosalam NA, El Aziz MHMA, Saad AS, Mohamed AW (2017) The Impact of Adjuvant Chemotherapy Initiation Time on the Outcome of Breast Cancer. J Oncol Transl Res 3: 113. doi: 10.4172/2476-2261.1000113

Copyright: (c) 2017 Mosalam NA, et al. This is an open-access article distributed under the terms of the Creative Commons Attribution License, which permits unrestricted use, distribution, and reproduction in any medium, provided the original author and source are credited. 
Citation: Mosalam NA, El Aziz MHMA, Saad AS, Mohamed AW (2017) The Impact of Adjuvant Chemotherapy Initiation Time on the Outcome of Breast Cancer. J Oncol Transl Res 3: 113. doi: 10.4172/2476-2261.1000113

Page 2 of 8

(stage III) if chemotherapy start was delayed for more than 60 days post-operatively [9].

A newly published study analyzed data for 24,843 patients with newly diagnosed stage I-III breast cancer from the California Cancer Registry found that starting chemotherapy within 30 to 60 days or 61 to 90 days after surgery did not adversely affect the survival odds but only patients who initiated chemotherapy 91 days or more postoperatively specially triple negative patients had a higher mortality hazard compared with those who started chemotherapy within 30 days of surgery [10].

Many oncology departments have waiting lists for patients starting chemotherapy as their number is steadily increase and often without coexistent increase in the needed resources, so such waiting lists cause worry among patients and since it will not be ethically acceptable to perform a trial of early vs late start of chemotherapy and to clarify this important clinical problem, we will evaluate the effect of time to chemotherapy (TTC) initiation on the overall survival and disease free survival in a large population-based study using the Ain shams educational hospital, oncology department database then it will be correlated with the data from other studies. In addition, we will trace the determinants of delayed chemotherapy administration once the causes are documented.

\section{Patients and Methods}

We identified 300 female patients at the department of clinical oncology and nuclear medicine, Ain Shams University, with stage I to III primary invasive breast cancer diagnosed between January 2007 till December 2011 to allow 5 year overall survival and disease free survival follow up, all patients underwent surgery and received adjuvant chemotherapy.

Patients with metastatic disease, double primary tumors or unknown stage at diagnosis were excluded; also patients treated with neoadjuvant chemotherapy or radiotherapy before chemotherapy were excluded as long radiotherapy waits will bias the results of this analysis

Patient information, including demographic characteristics, age, menopausal status, family history and whether the patient received radiation therapy.

\section{We obtained the following patient characteristics}

Clinical data such as, Patient's performance status at presentation, pathological stage at presentation according to American joint committee on cancer TNM criteria (Tables 1 and 2).

Surgical details such as, Date and type of breast surgery (mastectomy or breast conserving), presence of lymph node dissection and number of dissected lymph nodes (adequacy) was mentioned.

Tumor characteristics such as, Histopathological subtype, grading, nodal involvement, hormone receptor status, Her2/neu expression (Tables 3 and 4).

\section{Treatment Modalities Received Including}

Date of definitive surgery was defined as the date of the most extensive procedure ordinarily including axillary lymph node dissection.

Type of adjuvant chemotherapy and Time to chemotherapy (TTC) was defined as the number of weeks between definitive surgery and first administration of adjuvant chemotherapy and was categorized as the

\begin{tabular}{|l|c|c|}
\hline \multirow{2}{*}{ Age } & Mean \pm SD & No=300 \\
\hline \multirow{3}{*}{ Residence } & Rural & $49.59 \pm 9.91$ \\
\cline { 2 - 3 } & Urban & $25-75$ \\
\hline \multirow{2}{*}{ Menopausal state } & Rural & $230(76.70 \%)$ \\
\hline \multirow{2}{*}{ Family history } & NA & $1(23.00 \%)$ \\
\hline \multirow{2}{*}{ Type of surgery } & Pre & $148(49.30 \%)$ \\
\hline & Post & $152(50.70 \%)$ \\
\hline & Positive & $46(15.30 \%)$ \\
\hline & Negative & $254(84.70 \%)$ \\
\hline
\end{tabular}

Table 1: Patient characteristics.

\begin{tabular}{|c|c|c|c|}
\hline \multicolumn{2}{|c|}{ Pathological TNM Staging } & No. & $\%$ \\
\hline \multirow{4}{*}{$\mathrm{T}$} & $\mathrm{T} 1$ & 46 & $15.30 \%$ \\
\hline & T2 & 181 & $60.30 \%$ \\
\hline & T3 & 55 & $18.30 \%$ \\
\hline & $\mathrm{T} 4(\mathrm{~b})$ & 18 & $6.00 \%$ \\
\hline \multirow{4}{*}{$\mathrm{N}$} & NO & 95 & $31.70 \%$ \\
\hline & N1 & 88 & $29.30 \%$ \\
\hline & N2 & 61 & $20.30 \%$ \\
\hline & N3 & 56 & $18.70 \%$ \\
\hline \multirow{2}{*}{ No. of LN. } & Adequate & 273 & $91.00 \%$ \\
\hline & Inadequate & 27 & $9.00 \%$ \\
\hline \multirow{7}{*}{ Stage } & IA & 18 & $6.00 \%$ \\
\hline & IB & 1 & $0.30 \%$ \\
\hline & IIA & 75 & $25.00 \%$ \\
\hline & IIB & 69 & $23.00 \%$ \\
\hline & IIIA & 72 & $24.00 \%$ \\
\hline & IIIB & 18 & $6 \%$ \\
\hline & IIIC & 56 & $18.70 \%$ \\
\hline
\end{tabular}

Table 2: Clinico-pathological characteristics.

\begin{tabular}{|l|c|c|c|}
\hline \multicolumn{2}{|c|}{ Histopathology } & No. & $\%$ \\
\hline \multirow{2}{*}{ Type } & IDC & 279 & $93.00 \%$ \\
\hline \multirow{3}{*}{ Grade } & ILC & 21 & $7.00 \%$ \\
\hline \multirow{2}{*}{ LVI } & I & 3 & $1.00 \%$ \\
\hline \multirow{2}{*}{ PNI } & III & 269 & $89.70 \%$ \\
\hline & Positive & 28 & $9.30 \%$ \\
\hline & Negative/NA & 258 & $14.00 \%$ \\
\hline & Positive & 11 & $86.00 \%$ \\
\hline & Negative & 289 & $3.70 \%$ \\
\hline
\end{tabular}

Table 3: Histopathological characteristics.

\begin{tabular}{|l|c|c|c|}
\hline \multicolumn{2}{|c|}{ Tumor Biology } & No. & $\%$ \\
\hline \multirow{2}{*}{ ER } & Positive & 200 & $66.60 \%$ \\
\hline \multirow{3}{*}{ PR } & Negative & 100 & $33.30 \%$ \\
\hline \multirow{3}{*}{ HER2 } & Positive & 194 & $64.70 \%$ \\
\cline { 2 - 4 } & Negative & 106 & $35.30 \%$ \\
\hline & Positive & 49 & $16.30 \%$ \\
\cline { 2 - 4 } & Negative & 193 & $64.30 \%$ \\
\cline { 2 - 4 } & Equivocal & 26 & $8.70 \%$ \\
\cline { 2 - 4 } & Not available & 32 & $10.70 \%$ \\
\hline
\end{tabular}

Table 4: Hormonal receptor status, Her2 expression. 
Citation: Mosalam NA, El Aziz MHMA, Saad AS, Mohamed AW (2017) The Impact of Adjuvant Chemotherapy Initiation Time on the Outcome of Breast Cancer. J Oncol Transl Res 3: 113. doi: 10.4172/2476-2261.1000113

Page 3 of 8

following strata (less than 4 weeks, $4-8$ weeks, $>8$ weeks- $<12$ weeks, 12 weeks or more post-operatively.

Also data regarding Whether adjuvant hormonal treatment and Radiotherapy received or not, Onset of first relapse, site of first metastasis and Date of last follow up or death (Table 5).

\section{The endpoints of interest}

- $\quad$ 1ry endpoint: Overall survival; defined as the time in years from time of diagnosis to death or date of last contact.

- 2ry endpoint: Disease free survival; defined as the time in months between time of diagnosis and the first event including relapse (local, regional and/or distant) and contralateral invasive breast cancer. Relapse was documented as either a clinical, radiological or biopsy-proven disease relapse.

\section{Statistical Analysis}

The collected data was revised, coded, tabulated and introduced to a PC using Statistical package for Social Science (SPSS V20 for windows). Data was presented and suitable analysis was done according to the type of data obtained for each parameter.

\section{Descriptive statistics}

Mean, Standard deviation ( \pm SD), Minimum and maximum values (range) for numerical data, Frequency and percentage of nonnumerical data (Table 6).

\section{Analytical statistics}

- Chi-Square test was used to assess the relationship between the prognostic factors and different intervals to chemotherapy initiation. P-value: Statistical significance was defined as $\mathrm{P}<0.05$.

- Kaplan-Meier method was used to construct the Disease free survival and overall survival curves of their relationship with the different time intervals and the collected prognostic and predictive factors and compared via log-rank tests (Figures 1 and 2) (Table 7).

\section{Results}

A total of 300 female patients presented to the Clinical Oncology Department in the interval from January 2007 to December 2011 were enrolled in our study.

Along the study population, the age ranged from 25 years to 75

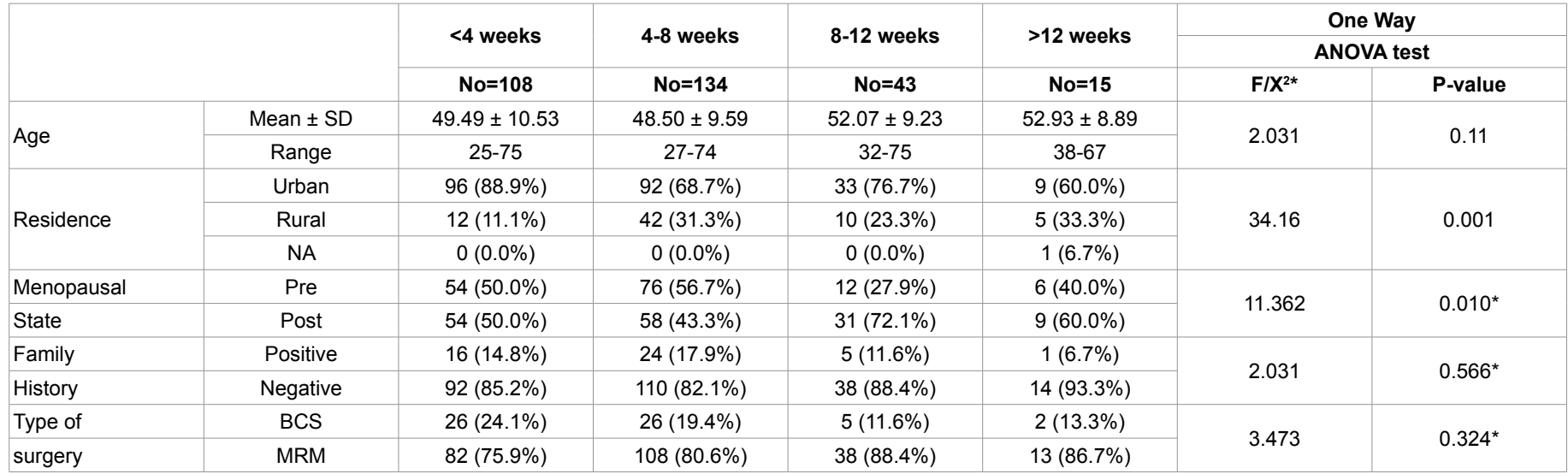

Table 5: Patient characteristics by interval from surgery to chemotherapy.

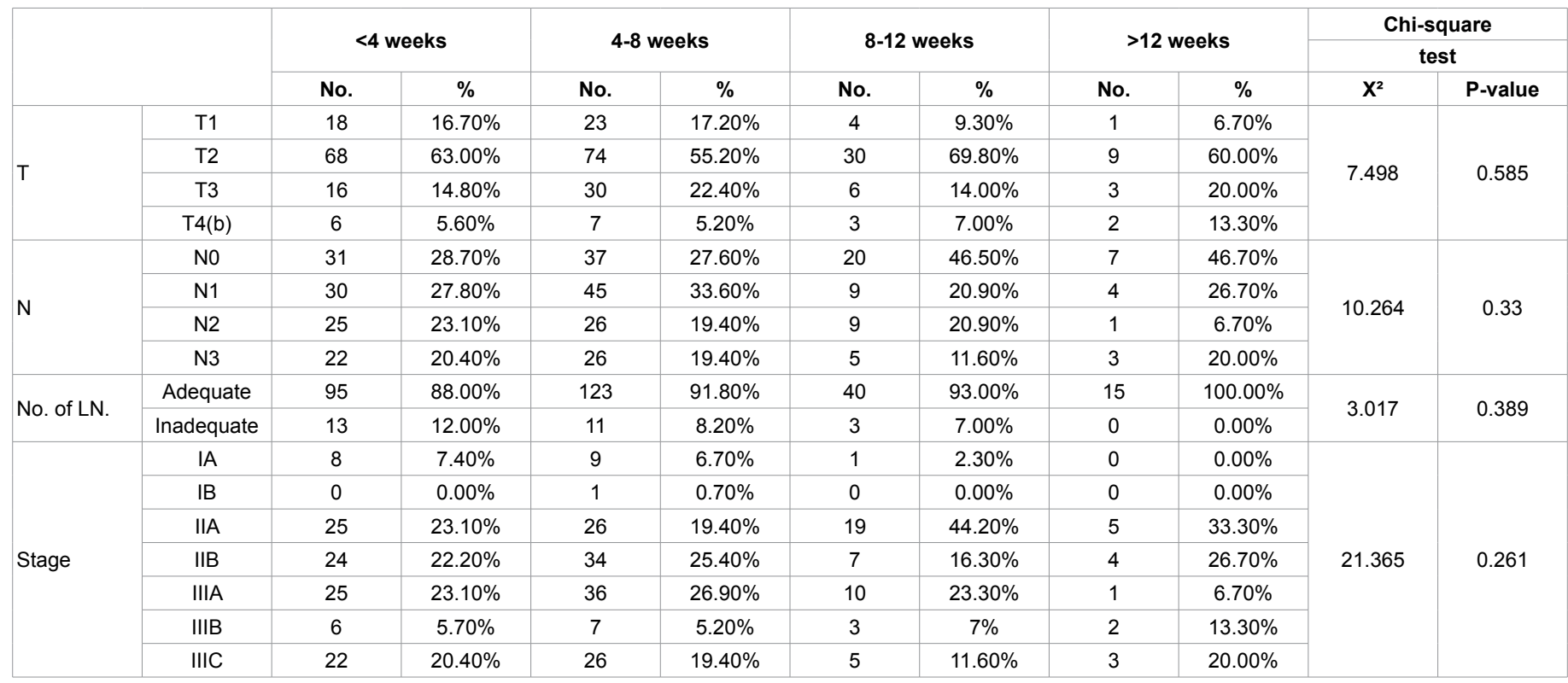

Table 6: Tumor characteristics distribution over the 4 different time groups. 
Citation: Mosalam NA, El Aziz MHMA, Saad AS, Mohamed AW (2017) The Impact of Adjuvant Chemotherapy Initiation Time on the Outcome of Breast Cancer. J Oncol Transl Res 3: 113. doi: 10.4172/2476-2261.1000113

Page 4 of 8

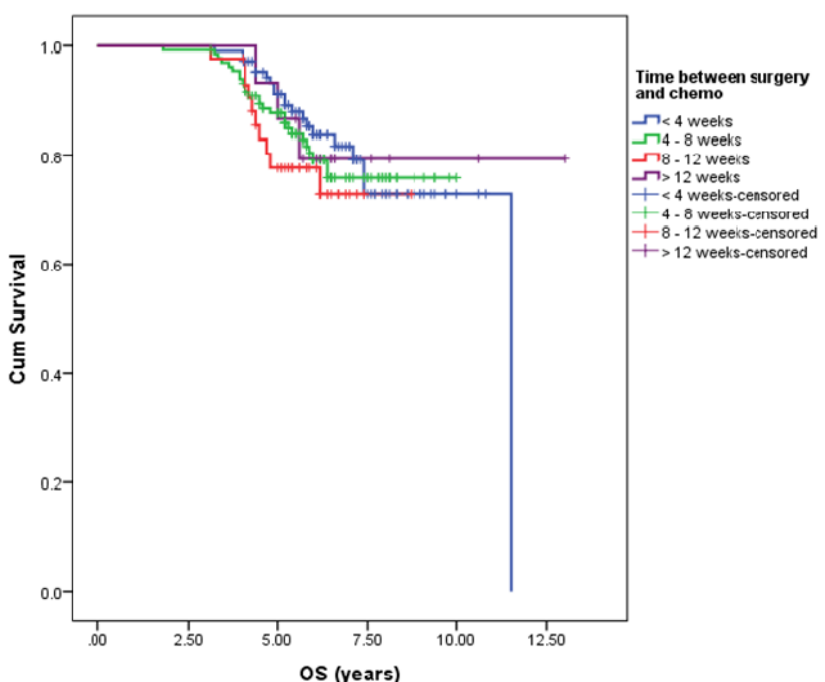

Figure 1: Overall survival plot according to interval between surgery and chemotherapy initiation using Kaplan-Meier method.

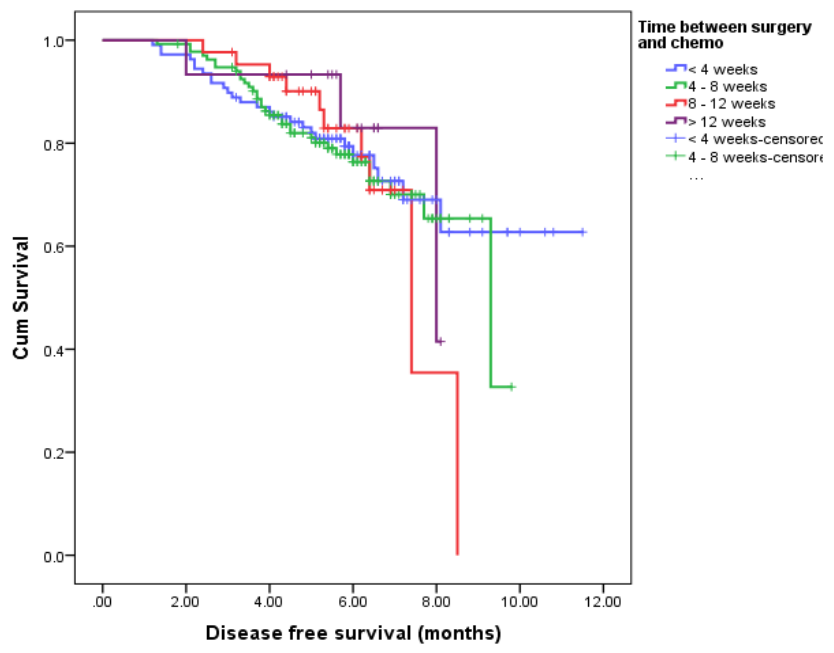

Figure 2: Breast cancer event-free survival according to interval between surgery and chemotherapy initiation using Kaplan-Meier method.

years with mean age at diagnosis was 50 years $(\mathrm{SD}=9.9)$ representing the majority of the patients (70\%).

Regarding stage at presentation, Stage II was the most common all through the 5 years follow up duration representing totally $48 \%$ of the cases. Yet, stage III represented $45 \%$ of the patients.

The median time to begin chemotherapy is 5 weeks $(\mathrm{SD}=2.8)$, ranging from 1-15 weeks. The patients were classified into 4 strata, a total of $36 \%$ of patients started chemotherapy in less than 4 weeks, 44.7\% waited $4-8$ weeks, $14 \%$ initiated their chemotherapy within $8-12$ weeks while only $5 \%$ were delayed more than 12 weeks to start their adjuvant chemotherapy.

We tried to trace the cause of chemotherapy delay, $50 \%$ of the delay was due to sociodemographic problems and long residential distance to institution as rural patients presented $33 \%$ of the patients in the $>12$ weeks group, while urban residence patients were mainly categorized

\begin{tabular}{|c|c|c|c|c|c|c|}
\hline & \multicolumn{3}{|c|}{ OS (years) } & \multicolumn{2}{|c|}{ Log rank test } \\
\hline & & \multirow{2}{*}{ No. } & \multirow{2}{*}{ Mean } & \multirow{2}{*}{$\begin{array}{c}\text { Standard } \\
\text { Error }\end{array}$} & \multirow{2}{*}{$\mathbf{X}^{2}$} & \multirow{2}{*}{ P-value } \\
\hline & & & & & & \\
\hline \multirow{2}{*}{ Residence } & Urban & 230 & 10.444 & 0.464 & \multirow{2}{*}{0.155} & \multirow{2}{*}{0.694} \\
\hline & Rural & 69 & 9.327 & 0.35 & & \\
\hline \multirow{2}{*}{ menopausal state } & Pre & 148 & 11.453 & 0.308 & \multirow{2}{*}{4.347} & \multirow{2}{*}{0.037} \\
\hline & Post & 152 & 9.485 & 0.305 & & \\
\hline \multirow{2}{*}{ Family history } & Negative & 254 & 11.163 & 0.254 & \multirow{2}{*}{2.606} & \multirow{2}{*}{0.106} \\
\hline & Positive & 46 & 8.999 & 0.687 & & \\
\hline \multirow{2}{*}{ Type of surgery } & BCS & 59 & 7.757 & 0.193 & \multirow{2}{*}{1.513} & \multirow{2}{*}{0.219} \\
\hline & MRM & 241 & 10.281 & 0.453 & & \\
\hline \multirow{4}{*}{$\mathrm{T}$} & $\mathrm{T} 1$ & 46 & 8.482 & 0.469 & & \\
\hline & $\mathrm{T} 2$ & 181 & 10.217 & 0.476 & 1515 & 0672 \\
\hline & T3 & 55 & 9.567 & 0.337 & 1.545 & $0.6 / 2$ \\
\hline & $\mathrm{T} 4(\mathrm{~b})$ & 18 & 7.412 & 0.314 & & \\
\hline & No & 95 & 11.259 & 0.388 & & \\
\hline & $\mathrm{N} 1$ & 88 & 9.781 & 0.373 & & \\
\hline IN & N2 & 61 & 9.431 & 0.352 & 0.549 & 0.900 \\
\hline & N3 & 56 & 8.208 & 0.306 & & \\
\hline Ne of IN & Adequate & 273 & 11.115 & 0.249 & 1717 & 10 \\
\hline NO. OT LIN. & Inadequate & 27 & 9.139 & 0.713 & 1.117 & 0.19 \\
\hline & IA & 18 & 5.5944 & 0.2191 & & \\
\hline & IB & 1 & 5.1 & . & & \\
\hline & IIA & 75 & 6.12 & 0.18627 & & \\
\hline STAGE & IIB & 69 & 6.2087 & 0.18653 & 1.284 & 0.973 \\
\hline & IIIA & 72 & 6.1056 & 0.21822 & & \\
\hline & IIIB & 18 & 6.4455 & 0.29244 & & \\
\hline & IIIC & 56 & 6.0224 & 0.19342 & & \\
\hline Tyne & IDC & 279 & 10.373 & 0.451 & 0324 & 057 \\
\hline Iуре & ILC & 21 & 9.143 & 0.457 & $0.0<4$ & 0.01 \\
\hline & 1 & 3 & 5.9 & 0.071 & & \\
\hline Grade & II & 269 & 10.428 & 0.456 & 0.681 & 0.711 \\
\hline & III & 28 & 8.419 & 0.445 & & \\
\hline IVI & $\mathrm{P}$ & 42 & 8.913 & 0.372 & 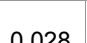 & 0866 \\
\hline LVI & $\mathrm{N}$ & 258 & 10.39 & 0.456 & $0.0<0$ & 0.000 \\
\hline PNU & $\mathrm{P}$ & 11 & 7.526 & 0.999 & 1407 & 0235 \\
\hline rivi & $\mathrm{N}$ & 289 & 10.464 & 0.453 & 1.401 & 0.200 \\
\hline ER & $\mathrm{P}$ & 194 & 10.422 & 0.477 & 0017 & 0820 \\
\hline ER & $\mathrm{N}$ & 106 & 9.185 & 0.279 & 0.041 & $0.0<0$ \\
\hline > > קח & $\mathrm{P}$ & 194 & 10.524 & 0.478 & م & 0 \\
\hline$r \pi$ & $\mathrm{N}$ & 106 & 9.049 & 0.293 & 0.020 & 0.000 \\
\hline & $\mathrm{P}$ & 49 & 9.002 & 0.411 & & \\
\hline HFR2 & $\mathrm{N}$ & 193 & 9.483 & 0.202 & 1385 & 0709 \\
\hline HER2 & EQI & 26 & 7.594 & 0.22 & 1.385 & 0.109 \\
\hline & Not available & 32 & 10.008 & 0.725 & & \\
\hline Rodinthoran & $P$ & 253 & 10.444 & 0.461 & 103 & 0718 \\
\hline Radotnerapy & $\mathrm{N}$ & 47 & 8.289 & 0.311 & 0.103 & 0.148 \\
\hline & P TAM & 192 & 10.784 & 0.485 & & \\
\hline Hormonal & PAI & 13 & 6.702 & 0.395 & 5.797 & 0.055 \\
\hline & $\mathrm{N}$ & 95 & 8.936 & 0.317 & & \\
\hline
\end{tabular}

Table 7: Kaplan-Meier method evaluating overall survival according to different patient's, tumor and therapeutic prognostic factors:

in the $<4$ weeks group and 4-8 group with a highly significant $P$ value $<0.0001$.

$20 \%$ of the cases were delayed 2ry to system related causes (late referral, time needed to get governmental insurance support and long waiting lists for diagnostic imaging, while other causes where 
Citation: Mosalam NA, El Aziz MHMA, Saad AS, Mohamed AW (2017) The Impact of Adjuvant Chemotherapy Initiation Time on the Outcome of Breast Cancer. J Oncol Transl Res 3: 113. doi: 10.4172/2476-2261.1000113

Page 5 of 8

\begin{tabular}{|c|c|c|c|c|c|c|}
\hline \multirow{2}{*}{ Parameters } & \multirow{2}{*}{ Groups } & \multicolumn{3}{|c|}{ Overall survival (years) } & \multicolumn{2}{|c|}{ Log rank test } \\
\hline & & Total N & Mean & SE & $\mathbf{X}^{2}$ & P-value \\
\hline \multirow{4}{*}{ Her $2+$ ve } & $<4$ weeks & 6 & 5.933 & 1.822 & \multirow{4}{*}{1.432} & \multirow{4}{*}{0.698} \\
\hline & 4-8 weeks & 14 & 5.5 & 1.201 & & \\
\hline & $8-12$ weeks & 3 & 6.233 & 0.651 & & \\
\hline & $>12$ weeks & 1 & 10.6 & 0 & & \\
\hline \multirow{4}{*}{ Triple negative } & $<4$ weeks & 14 & 8.309 & 0.277 & \multirow{4}{*}{3.541} & \multirow{4}{*}{0.316} \\
\hline & 4-8 weeks & 36 & 8.144 & 0.392 & & \\
\hline & $8-12$ weeks & 14 & 7.033 & 0.602 & & \\
\hline & $>12$ weeks & 5 & 7.36 & 0.662 & & \\
\hline \multirow{4}{*}{ Hormone positive } & $<4$ weeks & 81 & 9.755 & 0.404 & \multirow{4}{*}{1.012} & \multirow{4}{*}{0.798} \\
\hline & 4-8 weeks & 79 & 8.735 & 0.281 & & \\
\hline & 8-12 weeks & 25 & 6.762 & 0.252 & & \\
\hline & $>12$ weeks & 17 & 12 & 0.935 & & \\
\hline Stage III & $<4$ weeks & 52 & 9.003 & 0.435 & 2.185 & 0.535 \\
\hline
\end{tabular}

Table 8: Multivariable analysis for overall survival of the patients according to their molecular subtype and high risk features according to the time to chemotherapy categories.

\begin{tabular}{|c|c|c|c|c|c|c|}
\hline \multirow{3}{*}{ ParameterS } & \multirow{3}{*}{ Groups } & \multirow{2}{*}{\multicolumn{3}{|c|}{$\begin{array}{c}\text { Disease free survival } \\
\text { (Months) }\end{array}$}} & \multirow{2}{*}{\multicolumn{2}{|c|}{ Log rank test }} \\
\hline & & & & & & \\
\hline & & Total $\mathbf{N}$ & Mean & SE & $\mathbf{X}^{2}$ & P-value \\
\hline \multirow{4}{*}{ Her $2+$ ve } & $<4$ weeks & 6 & 6.04 & 0.552 & \multirow{4}{*}{0.838} & \multirow{4}{*}{0.841} \\
\hline & 4-8 weeks & 14 & 6.107 & 0.552 & & \\
\hline & 8-12 weeks & 3 & 5.8 & 0.45 & & \\
\hline & $>12$ weeks & 1 & 5.7 & 0 & & \\
\hline \multirow{4}{*}{ Triple negative } & $<4$ weeks & 14 & 6.486 & 0.315 & \multirow{4}{*}{3.715} & \multirow{4}{*}{0.294} \\
\hline & 4-8 weeks & 36 & 5.675 & 0.233 & & \\
\hline & 8-12 weeks & 14 & 5.671 & 0.422 & & \\
\hline & $>12$ weeks & 5 & 6.36 & 0.59 & & \\
\hline \multirow{4}{*}{ Hormone positive } & $<4$ weeks & 81 & 9.135 & 0.478 & \multirow{4}{*}{0.729} & \multirow{4}{*}{0.866} \\
\hline & 4-8 weeks & 79 & 8.351 & 0.315 & & \\
\hline & 8-12 weeks & 25 & 6.788 & 0.225 & & \\
\hline & $>12$ weeks & 17 & 7.25 & 0.992 & & \\
\hline \multirow{4}{*}{ Stage III } & $<4$ weeks & 52 & 8.103 & 0.539 & \multirow{4}{*}{0.508} & \multirow{4}{*}{0.917} \\
\hline & 4-8 weeks & 70 & 7.541 & 0.384 & & \\
\hline & 8-12 weeks & 18 & 6.131 & 0.351 & & \\
\hline & $>12$ weeks & 6 & 7.083 & 0.928 & & \\
\hline \multirow{3}{*}{ Grade III } & $<4$ weeks & 13 & 5.997 & 0.458 & \multirow{3}{*}{0.55} & \multirow{3}{*}{0.759} \\
\hline & 4-8 weeks & 10 & 6.611 & 1.138 & & \\
\hline & 8-12 weeks & 5 & 5.9 & 0.433 & & \\
\hline
\end{tabular}

Table 9: Multivariable analysis for disease free survival of the patients according to their molecular subtype and high risk features according to the time to chemotherapy categories. variable, between postoperative wound complications or late recovery $(1.7 \%)$ and comorbidities (1\%), positive margins and re-surgery (1\%), late presentation (1.3\%) and patient's incompliance and missed appointments (1\%) while the delay causes were unreported in $24 \%$ of the patients.

Patient's characteristics were stratified by time to chemotherapy groups and have been evaluated by the $\mathrm{X}^{2}$ test, showing that positive family history was found mainly in the subgroups of patients started their chemotherapy in less than 4 weeks and the subgroup of patients with chemotherapy initiation from 4-8 weeks with percentages $14.8 \%$ and $17.9 \%$, respectively but yet, the statistical association wasn't found $(P=0.566)$. Also delays in chemotherapy have been reported to be more likely to occur in postmenopausal (elderly) patients with significant $\mathrm{P}$ value $=0.01$ mainly secondary to multiple comorbidities and long needed post-operative recovery interval.

Survival analysis using Kaplan- Meier method for overall survival (OS) according to TTC demonstrated, the 5 year overall survival estimate was $91 \%, 87.7 \%, 77.7 \%, 86.7 \%$ among patients who received chemotherapy $<4$ weeks, $4-<8$ weeks, $8-<12$ weeks and 12 weeks or more after surgery, respectively.

Non-significant association was observed according to the timing of adjuvant chemotherapy initiation with P-value 0.67. Indicating the absence of any hazard for delaying the start of chemotherapy up to 12 weeks postoperative.

The analyses have also been performed for DFS, that the 5 year follow up resulted in Disease free survival rates $82 \%, 81 \%, 90 \%$, and $83 \%$ ( $\log$-rank $\mathrm{P}=0.94$ ) for groups 1 through 4 , respectively. The DFS curves expressed the same insignificant pattern as the OS curves regardless the TTC categories with (P value $=0.9$ ).

As expected, worse DFS was associated with large tumor size (T3, $\mathrm{T} 4 \mathrm{~b}$ ), greater lymph node involvement and locally advanced stage III (B and $C)$ with a significant $P$ value $(0.03,0.01$ and 0.0001 , respectively).

As regards molecular subtypes versus DFS, a highly significant worse outcome with HER2 enriched and triple negative subtypes with significant $P$ value 0.04 and 0.002 , respectively, While hormone positive subtype demonstrated a better DFS $(\mathrm{P}=0.01)$.

In a subgroup multivariate analysis between breast cancer molecular subtypes and the OS along the TTC categories, no overall survival impact for chemotherapy initiation longer than 12 weeks postoperative

\begin{tabular}{|c|c|c|c|c|c|c|c|c|c|c|c|}
\hline \multirow{2}{*}{\multicolumn{2}{|c|}{ Parameters }} & \multicolumn{2}{|c|}{$<4$ weeks } & \multicolumn{2}{|c|}{ 4-8 weeks } & \multicolumn{2}{|c|}{ 8-12 weeks } & \multicolumn{2}{|c|}{$>12$ weeks } & \multicolumn{2}{|c|}{ Chi-square test } \\
\hline & & No. & $\%$ & No. & $\%$ & No. & $\%$ & No. & $\%$ & $\mathbf{X}^{2}$ & P-value \\
\hline \multirow{2}{*}{ HER2 only } & No & 102 & $94.40 \%$ & 120 & $89.60 \%$ & 40 & $93.00 \%$ & 14 & $93.30 \%$ & \multirow{2}{*}{2.065} & \multirow{2}{*}{0.559} \\
\hline & Yes & 6 & $5.60 \%$ & 14 & $10.40 \%$ & 3 & $7.00 \%$ & 1 & $6.70 \%$ & & \\
\hline \multirow{2}{*}{$\begin{array}{l}\text { Triple } \\
\text { negative }\end{array}$} & No & 94 & $87.00 \%$ & 98 & $73.10 \%$ & 29 & $67.40 \%$ & 10 & $66.70 \%$ & \multirow{2}{*}{10.397} & \multirow{2}{*}{0.015} \\
\hline & Yes & 14 & $13.00 \%$ & 36 & $26.90 \%$ & 14 & $32.60 \%$ & 5 & $33.30 \%$ & & \\
\hline Hormone & No & 27 & $25.00 \%$ & 55 & $41.00 \%$ & 18 & $41.90 \%$ & 7 & $46.70 \%$ & \multirow{2}{*}{8.554} & \multirow{2}{*}{0.036} \\
\hline positive & Yes & 81 & $75.00 \%$ & 79 & $59.00 \%$ & 25 & $58.10 \%$ & 17 & $53.30 \%$ & & \\
\hline \multirow{2}{*}{ Stage III } & No & 58 & $53.70 \%$ & 69 & $51.50 \%$ & 27 & $62.80 \%$ & 9 & $60.00 \%$ & \multirow{2}{*}{1.887} & \multirow{2}{*}{0.596} \\
\hline & Yes & 52 & $46.30 \%$ & 70 & $48.50 \%$ & 18 & $37.20 \%$ & 6 & $40.00 \%$ & & \\
\hline \multirow{2}{*}{ Grade III } & No & 95 & $88.00 \%$ & 124 & $92.50 \%$ & 38 & $88.40 \%$ & 15 & $100.00 \%$ & \multirow{2}{*}{3.299} & \multirow{2}{*}{0.348} \\
\hline & Yes & 13 & $12.00 \%$ & 10 & $7.50 \%$ & 5 & $11.60 \%$ & 0 & $0.00 \%$ & & \\
\hline
\end{tabular}

Table 10: Relation between time to chemotherapy initiation and the breast cancer subtypes and high risk patient's distribution: 
for all breast cancer subtypes even triple negative patients with nonsignificant $P$ value $=0.3$.

The same correlation was made as regard the DFS and yielded the same lack of association where the correlation resulted into insignificant $P$ values for HER2 enriched, triple negative and hormonal positive tumors with values $0.8,0.2$ and 0.8 , respectively (Tables 8,9 and 10).

\section{Discussion}

In this study, we observed that a delay in chemotherapy initiation up to 12 weeks postoperatively for all breast cancer subtypes even triple negative patients have similar overall survival and disease free survival with non-significant $\mathrm{P}$ values.

Our data are unique and adds to the available literature as it is one of the first reports on the impact of both treatment delay and completion of chemotherapy on treatment outcomes, as it was available to report the number of cycles of chemotherapy received for each patient from our registry data ensuring that each patient included had completed 6 months of adjuvant chemotherapy regardless of its type, making the results of the study reliable and eliminated the controversy about how many cycles have been received, adding also the type of hormonal treatment and its duration so that allowed us to eliminate any bias of the results as regards the affection on the treatment outcomes.

This study's point of strength is being the first Egyptian study using that large sample size collected from a tertiary center data registry which is rich by clinical and treatment information regarding each patient, reflecting general practice pattern allowing generalization of our results specially because of the recent years of inclusion guarantying the use of contemporary systemic regimens.

Our study's median time to initiate adjuvant chemotherapy was 5 weeks $(\mathrm{SD}=2.8)$ and that time frame is supported by the quality measures by the (ASCO) and (NCCN) organizations, recommending that adjuvant chemotherapy should be started within 120 days of diagnosis especially for women aged less than 70 years with nonmetastatic breast cancer [8].

Similar time to chemotherapy ( 5.8 weeks) was found by Fedewa et al. [11] which addressed the related factors causing chemotherapy delay among 107,587 African American and Hispanic patients with invasive breast cancer (stages I to III) using the National Cancer Database.

Longer median time was found in a study conducted by Vandergrift et al. [12] that assessed time from pathological diagnosis to start of chemotherapy among 6,622 women with stage I-III breast cancer that were treated among nine NCCN centers, where the median TTC was 11.3 weeks.

Most of Our patients (44.7\%) started their chemotherapy within 4-8 weeks while only $5 \%$ were delayed more than 12 weeks to start their adjuvant chemotherapy and this distribution was quite close to the Danish Breast Cancer Cooperative Group (DBCG) study on 7,501 patients by Cold et al. [13] which assessed the survival effect of delaying adjuvant chemotherapy start in patients with early breast cancer for up to 3 months after surgery as $50 \%$ of the studied population started their chemotherapy within 4-8 weeks while those waited more than 12 weeks didn't exceed 3-4\%.

The main determinant of delayed chemotherapy start in the current study was sociodemographic in nature as rural patients were the most population suffered from delay and that is surely explained by taking this database from a tertiary center hospital that receives patients from several referring centers across Egypt, while the second ranked cause of delay was system related causes as late referrals, long time needed to get governmental insurance support and long waiting lists for diagnostic imaging, the same causes were described by Vandergrift et al. [12] who mainly investigated this question across Nine institutions participating in the NCCN database including City of Hope Comprehensive Cancer Center, the university of Texas MD Anderson Cancer Center, University of Michigan and others finding that system level factors related to transferring care, insurance issues, repeat of diagnostic investigations and far residence from the treating institution were the main causes of TTC prolongation.

Among our patient's characteristics associated with delayed chemotherapy, being a post-menopausal, older age patient with multiple comorbidities were a significant factors for delay and that's coincides with a recent study results by Chavez-Mac Gregor et al. [10] which used data from the California Cancer Registry finding that older age, reconstructive surgery and sociodemographic factors were the main obstacles leading to longer TTC.

Downing et al. [14] agreed with our results as regards old age is a consistent factor of postoperative chemotherapy delay using a retrospective observational Study over 11,054 women with invasive breast cancer.

Ninety five percent $(95 \%)$ of our study population started their adjuvant chemotherapy within 12 weeks; we found that the 5 year OS and DFS were statistically similar along the different time categories and this is in agreement with the ASCO trial conducted using Data from British Columbia by Lohrisch et al. [15] that was a retrospective study held over 2,594 patients with stage I-II breast cancer diagnosed between 1989-1998 in that patients were assigned in terms of time (weeks) from definitive curative surgery to start of adjuvant chemotherapy similar to our study design ( $<4$ weeks, $>4-8$ weeks, $>8$ to 12 weeks and $>12$ weeks) assessing the overall survival and relapse free survival, confirming that RFS and OS were similar for women starting chemotherapy up to 12 weeks after surgery without any adverse outcomes.

Using Kaplan-Meier method for overall survival (OS) analysis according to TTC demonstrated that 5 year overall were $91 \%, 87.7 \%$, $77.7 \%, 86.7 \%$ among patients whom received chemotherapy $<4$ weeks, $4-<8$ weeks, $8-<12$ weeks and 12 weeks or more after surgery, respectively, that is quite close to Lohrisch et al.s [15] percentages as the five-year OS rates were $84 \%, 85 \%, 89 \%$ for the same time categories, respectively.

Our DFS rates after 5 years follow up were $82 \%, 81 \%, 90 \%$ and $83 \%$ for groups 1 through 4 , respectively. Which were higher than that by Lohrisch et al. [15] that represented $74 \%, 79 \%, 82 \%$ and $69 \%$ for patients starting chemotherapy 4 weeks or fewer, more than 4 to 8 weeks, more than 8 to 12 weeks.

Contradictory to our results, Lohrisch et al. [15] found that patient's DFS and OS appear to be compromised by delays of more than 12 weeks-24 weeks after definitive surgery with significant $P$ values ( $P=0.004, P=0.004$, respectively) and that maybe became achievable because of the large sample size of the study overall and specially in (>12 weeks) time category.

We could not find any significance as regard the effect of delay beyond 12 weeks on the overall survival or recurrence and that is surely explained by being $95 \%$ of the patients started their adjuvant chemotherapy within 3 months after surgery and only $5 \%$ of patients experienced more than 12 weeks delay in chemotherapy initiation 
making this category sample size quite small to be correlated with the treatment outcomes and thus be matched in terms of significance found in the previous mentioned study.

Our results coincides also with other study conducted by the Danish Breast Cancer Cooperative Group (DBCG) by Cold et al. [13] which assessed the effect of delaying the start of adjuvant chemotherapy for early breast cancer for up to 3 months postoperatively on the overall survival over 7,501 patients treated between 1977 and 1999 , they divided their study population into four strata (1-3, 4, 5 and 6-13 weeks) founding no significant survival benefits among patients with delayed chemotherapy till 13 weeks after surgery with P-values ranging from 0.2 to 0.7 .

We confirmed This lack of an association and insignificance between TTC and OS in multivariate analyses taking known high risk prognostic factors into account (high grade tumors, advanced stage (III), triple negative subtype) where similar outcomes along the time categories were obtained and no survival or recurrence adverse effect differences resulted, this was quit equal to the results founded by Cold et al. [13] study yielding the same insignificance, confirming that the prognosis for patients starting chemotherapy within 3 weeks was quite similar to those starting chemotherapy up to 13 weeks postoperatively (Table 10).

A more recent ASCO study was conducted by Gagliato et al. [9] on the overall survival, distant-relapse-free survival outcomes according to TTC over 6,827 patients. Overall it agreed with our results as regards the indifferences in terms of OS and DFS between cohorts starting chemotherapy either within 0 to 30 days, 31 to 60 days, or more than 60 days postoperatively.

Gagliato et al. [9] was contradictory to our findings as he showed a significance only with more advanced stage disease who experienced worse outcome in terms of RFS and DRFS (stage II and III) and OS (stage III) if chemotherapy start was delayed for more than 60 days postoperatively and that can be explained by the patients characteristics included in this study, as grade III tumors represented 53\% of the studied population in contrast to ours in which represented only $9.3 \%$ of the total population which are known adverse feature in breast cancer affecting the overall patient's prognosis. Also, patients with triplenegative disease $(17 \%)$ and those with HER2-positive disease (16\%) who were treated with Herceptin, had an inferior OS if chemotherapy started longer than 60 days after surgery, while no significance were seen in terms of RFS or DRFS unlike our results as regards the lack of significance in both triple negative (23\%) and HER2 subtypes (8\%) in terms of OS or DFS, that can be justified by the larger HER2 positive sample size (16\%) in contrast to ours which was only $8 \%$.

A recent study on Chavez-Mac Gregor et al. [10] analyzed data for 24,843 patients with newly diagnosed stage I-III breast cancer during 2005-2010 from the California Cancer Registry, Patients were found to be distributed as $(21.0 \%, 50 \%, 19.2 \%, 9.8 \%)$ according to Time of chemotherapy start within (30 days, 31 to 60 days, 61 to 90 days and 91+ days, respectively), it supported our findings as starting chemotherapy within 30 to 60 days or 61 to 90 days after surgery did not adversely affect the survival odds but again it contradicted our results as it found that Patients who initiated chemotherapy 91 days or more postoperatively specially triple negative patients had a higher mortality hazard compared with those who started chemotherapy within 30 days of surgery.

We found no difference in OS or DFS in hormone receptor (ER, PR) positive and HER2 subgroups along the different TTC categories. This was consistent with Chavez-Mac Gregor et al's [10] study which agreed with the non-significance of delayed chemotherapy as regard both OS and DFS till more than 91 days among those with hormone receptor positive (Hazard ratio, 1.25) or HER2+ (Hazard ratio, 1.28) tumors.

As regards the non-inferiority of OS or DFS in triple negative patients along different TTC groups, supported our findings, as she evaluated whether delays from diagnosis to initial treatment in triple negative breast cancer (TNBC) only impacts survival or loco-regional recurrence over 301 patients where patients were divided into three groups based on interval to treatment: $0-45,46-90$, and $>90$ days confirming that the Interval to treatment did not impact overall survival $(p=0.24)$ or locoregional recurrence $(p=0.54)$.

A Malaysian study conducted by Mujar et al. [16] also correlated between survival of 648 patients with invasive breast cancer who are treated between 2004-2005 and time to primary treatment (TPT) whether it was surgery or chemotherapy showing that Delayed TPT up to 2 months did not affect overall survival on univariate and multivariate analyses agreeing with our study conclusions.

Moreover, the safety time frame till 12 weeks for administration of the adjuvant chemotherapy in breast cancer is assessed by Systematic search of the Literature conducted across five electronic databases: PubMed, EMBASE, CINAHI, Scopus and Science Direct with a total of 33 articles included in this evidence based systematic review, that comprised of 255,366 observations concluding that the initiation of chemotherapy for breast cancer should be within 90 days after diagnosis to enhance the quality of life for breast cancer patients [17].

Our study has some limitations because of its retrospective nature but any randomized controlled trial comparing the effect chemotherapy initiation delays on survival after surgery would not be feasible for obvious ethical reasons. Therefore, the question of timing to CT cannot be answered prospectively.

\section{Conclusion}

Based on currently available data, including the findings in this study, we would suggest that all breast cancer patients whom are candidates for adjuvant chemotherapy should receive their chemotherapy within 12 weeks of surgery.

\section{References}

1. Fkih IM, Privat M, Ponelle F, Penault-Llorca F, Kenani A, et al. (2015) Identification of miR-10b, miR-26a, miR-146a and miR-153 as potential triplenegative breast cancer biomarkers. Cell Oncol 38: 433-442.

2. Ibrahim AS, Khaled HM, Nabiel NH (2014) Cancer Incidence in Egypt Results of the National Population-Based Cancer Registry Program. J Cancer Epidemiol 2014: 18 Pages.

3. Early Breast Cancer Trialists' Collaborative Group (EBCTCG) (2005) Effects of chemotherapy and hormonal therapy for early breast cancer on recurrence and 15 year survival: An overview of the randomised trials. Lancet 365: 1687-1717.

4. Unger-Saldaña $K(2014)$ Challenges to the early diagnosis and treatment of breast cancer in developing countries. World J Clin Oncol 5: 465-477.

5. Caplan L (2014) Delay in breast cancer: Implications for stage at diagnosis and survival. Front Public Health 2: 87-16

6. Bonadonna G, Valagussa P, Moliterni A, Zambetti M, Brambilla C (1995) Adjuvant cyclophosphamide, methotrexate and fluorouracil in node-positive breast cancer: The results of 20 years of follow-up. N Engl J Med 332: 901-926.

7. Richardson LC, Royalty J, Howe W, Helsel W, Kammerer W, et al. (2010) Timeliness of breast cancer diagnosis and initiation of treatment in the National Breast and Cervical Cancer Early Detection Program, 1996-2005. Am J Public Health 100: 1769-1776.

8. Desch CE, McNiff KK, Schneider EC, McClure J, Lepisto E, et al. (2008) 
Citation: Mosalam NA, El Aziz MHMA, Saad AS, Mohamed AW (2017) The Impact of Adjuvant Chemotherapy Initiation Time on the Outcome of Breast Cancer. J Oncol Transl Res 3: 113. doi: 10.4172/2476-2261.1000113

Page 8 of 8

American Society of Clinical Oncology/National Comprehensive Cancer Network quality measures. J Clin Oncol 26: 3631-3637.

9. Gagliato DM, Gonzalez-Angulo AM, Lei X, Theriault RL, Giordano SH, et al. (2014) Clinical impact of delaying initiation of adjuvant chemotherapy in patients with breast cancer. J Clin Oncol 32: 735-744.

10. Chavez-Mac Gregor M, Christina CA, Lichtensztajn DY and Giordano SH (2016) Delayed initiation of adjuvant chemotherapy among patients with breast cancer. JAMA Oncol 2: 322-329.

11. Fedewa SA, Ward EM, Stewart AK and Edge SB (2010) Delays in adjuvan chemotherapy treatment Among patients with breast cancer are more likely in african american and hispanic populations: A National Cohort Study 20042006. J Clin Oncol 28: 4135-4141.

12. Vandergrift JL, Niland JC, Theriault RL, Edge SB, Wong YN, et al. (2013) Time to adjuvant chemotherapy for breast cancer in National comprehensive cancer network institutions. J Natl Cancer Inst 105: 104-112
13. Cold S, During M, Ewertz M, Knoop A, Møller S (2005) Does timing of adjuvant chemotherapy influence the prognosis after early breast cancer? Results of the Danish Breast Cancer Cooperative Group (DBCG). Br J Cancer 93: 627-632.

14. Downing A, Twelves C, Forman D, Lawrence G, Gilthorpe (2014) Time to begin adjuvant chemotherapy and survival in breast cancer patients: A retrospective observational study using latent class analysis. Breast J 7: 93-97.

15. Lohrisch C, Paltiel C, Gelmon K, Speers C, Taylor, S et al. (2006) Impact on survival of time from definitive surgery to initiation of adjuvant chemotherapy for early-stage breast cancer. J Clin Oncol 24: 4888-4894.

16. Mujar M, Dahlui M, Yip CH, Taib NA (2013) Delays in time to primary treatment after a diagnosis of breast cancer: Does it impact survival? Prev Med 56: 222 224

17. Williams $F$ (2015) Assessment of breast cancer treatment delay impact on prognosis and survival: A look at the evidence from systematic analysis of the literature. A pilot study. J Cancer Biol Res 3: 1071. 